\title{
La gens Calpurnia de Barcino
}

\author{
Isabel RODÀ
}

(ICAC-UAB)

\section{Resumen}

El hallazgo de un nuevo pedestal en Barcino dedicado al magistrado municipal Quinto Calpurnio Flavo, permite establecer las relaciones familiares con otros miembros ya conocidos de la gens y al mismo tiempo precisar la datación de los pedestales en función de la presentación formal del texto y asimismo corroborar el peso en la vida ciudadana de ciertos "lobbies", de los libertos y la de la movilidad social en Barcino.

Palabras Clave: Epigrafía, Barcino, Sociedad romana.

\section{Summary}

The finding of a new pedestal in Barcino dedicated to the municipal magistrate Quintus Calpurnius Flavus, allows us to establish relationships with other family members and acquaintances of the gens. It also clarifies the dating of the pedestals in terms of the formal presentation of the text, and corroborates the influence of certain "lobbies" and freedman in public life as well as social mobility.

Keywords: Epigraphy, Barcino, Roman Society.

En la rica epigrafía de Barcino son bien conocidos los componentes de la familia Calpurnia. Los miembros más eminentes, magistrados municipales, nos son conocidos por una placa de finales del siglo I d.C. (IRC IV, 56) y dos pedestales, uno publicado desde antiguo $(I R C I V, 55)$ y otro inédito de cuyo texto vamos tratar aquí.

En los dos primeros monumentos (IRC IV, 55-56) constan las relaciones familiares. E1 pedestal (fig. 1) nos informa de que fue erigido por la madre del magistrado municipal, Cominia Nereis, que tiene un nomen poco conocido en Hispania oriental, pero en cambio presente en la vecina Narbonense, con la que Barcino mantuvo estrechos contactos ${ }^{1}$. Por otra parte, el helénico Nereis tampoco es demasiado frecuente en la península Ibérica y es propio más bien de mujeres esclavas o libertas ${ }^{2}$.
1 M. GAYRAUD, Narbonne antique des origines à la fin $d u$ IIIè siècle, París 1981, pp, 565, 567, I. RODÀ, "La escultura del sur de la Narbonense y el norte de Hispania Citerior: paralelos y contactos", Actas de la III Reunión sobre escultura romana en Hispania (Córdoba 1997), P. León, T. Nogales edd., Madrid 2000, pp.173-196; EAD., "Imago mortis: el componente itálico en el mundo funerario de Barcino y Tarraco", Mors omnibus instat, (Tudela
2009), J. Andreu, D. Espinosa, S. Pastor coord., Madrid 2011, pp. 233-254, esp. pp. 241-247.

2 J.M. ABASCAL, Los nombres personales en las inscripciones latinas de España, Anejos de Antigüedad y Cristianismo 1, Murcia 1994, pp. 115-116; H. SOLIN, Die grieschischen Personennamen in Rom. Ein Namenbuch, $2^{\mathrm{a}}$ ed., Berlín-Nueva York 2003, pp. 427-428. 


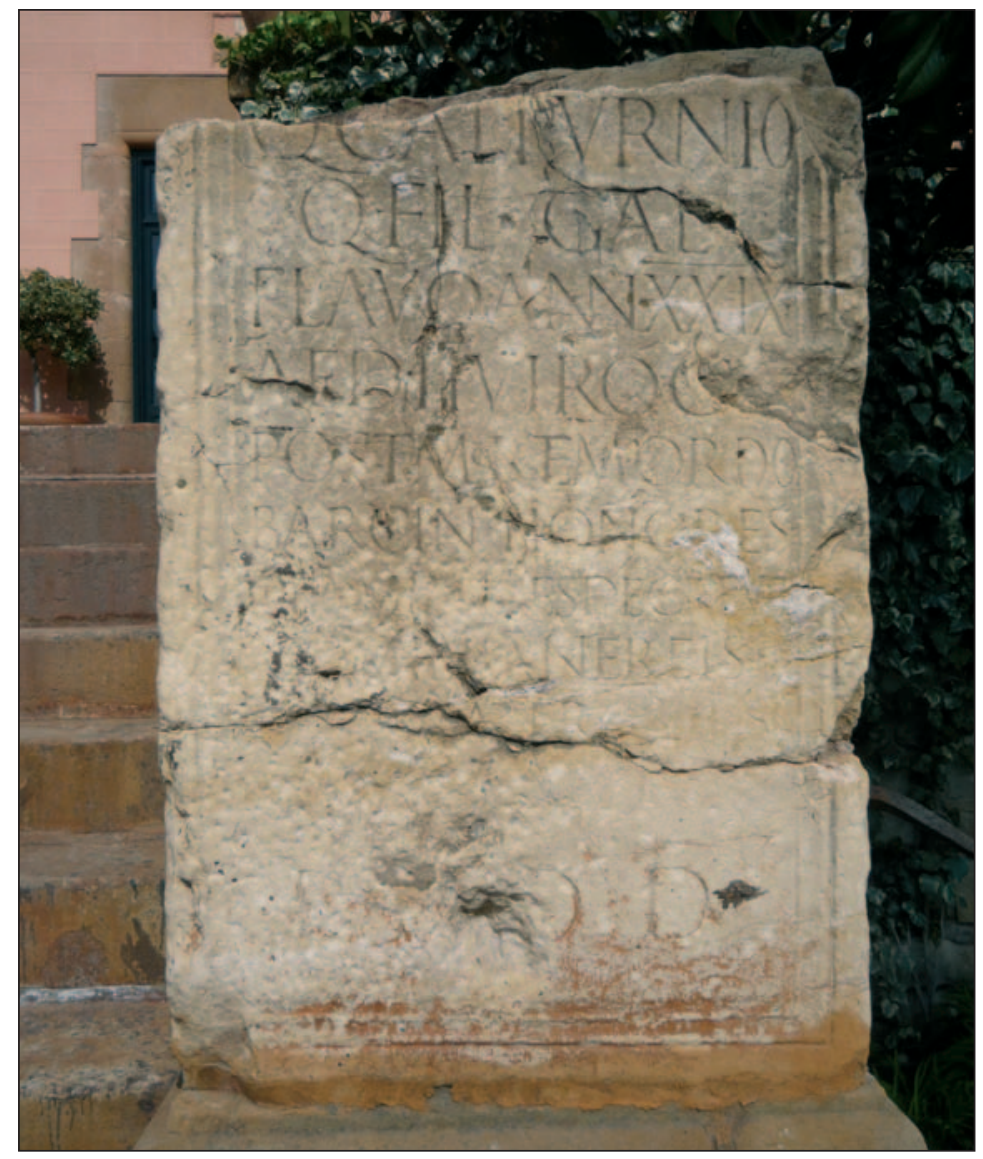

Figura 1.- IRC IV, 55 en su ubicación actual en el jardín de la Torre dels Lleons

(Esplugues de Llobregat). Foto I. Rodà.

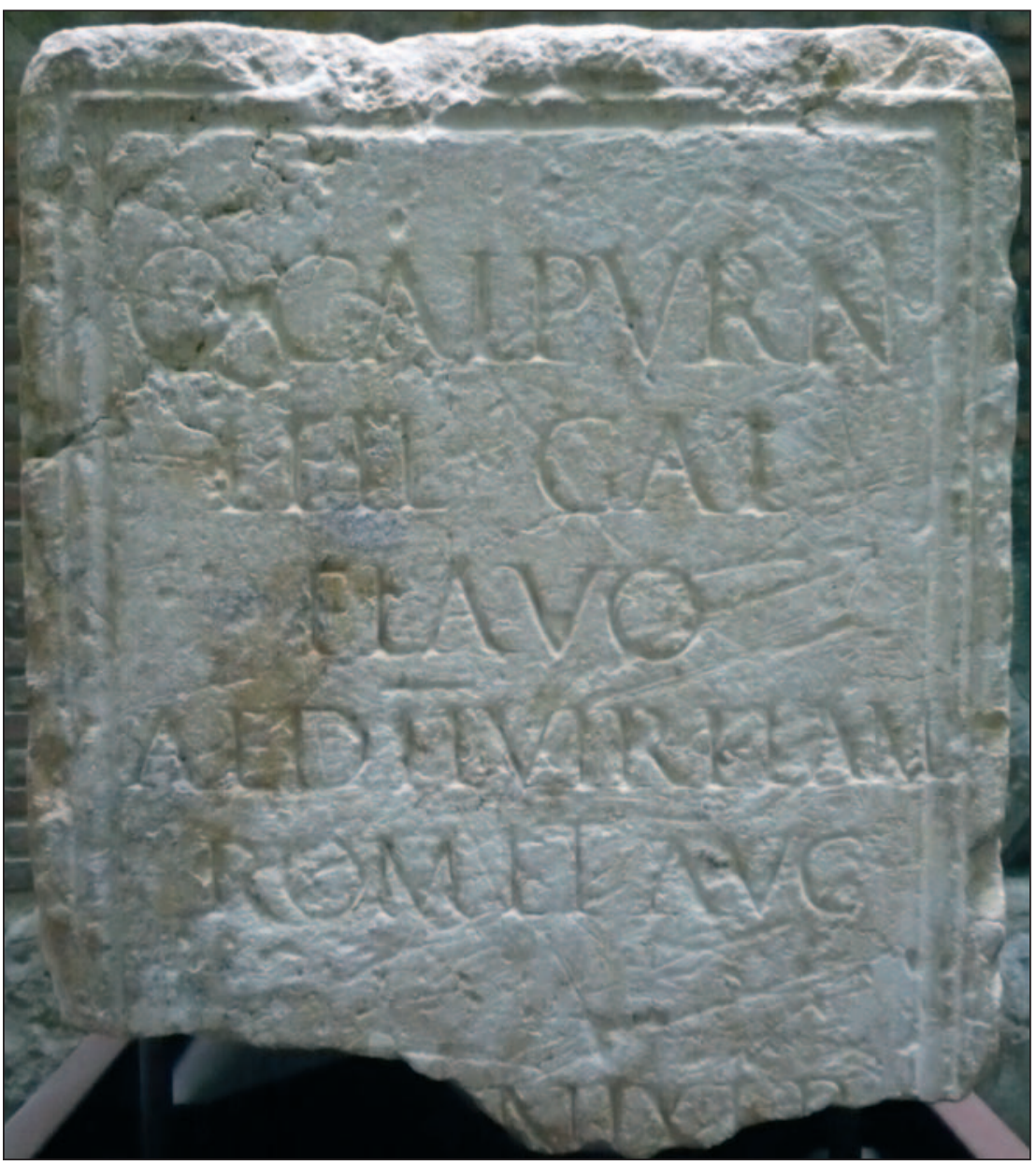

Figura 2.- Pedestal descubierto en la iglesia de Santa Maria del $\mathrm{Pi}$, ahora en el recinto del templo romano.

Foto I. Rodà 
También en el epígrafe, hoy perdido (IRC IV, 56, fig. 3), se nos informa sobre Lucius Calpurnius Iuncus, con todos los grados de la carrera culminados y padre de otro personaje homónimo, también brillante magistrado que repitió por dos veces el cargo de duumvir. En cambio la esposa, una Valeria, consta explícitamente sin cognomen y como liberta de Lucius. El dibujo de Accursius nos informa que el campo epigráfico estaba contorneado por una moldura, pero más que pensar en un pedestal, el listado de nombres que parece haberse conservado completo induce a pensar en una placa funeraria para ser inserta en un monumento funerario de cierta entidad.

Una vez más comprobamos la permeabilidad de la sociedad barcinonense y el activo papel de los libertos en el día a día de la colonia de Barcino y que el culto imperial fue una institución de gran vitalidad para la promoción y movilidad social ${ }^{3}$.

Lo continuamos comprobando en el resto de las inscripciones de la gens Calpurnia ya que todas ellas, en número de cuatro, home- najean a libertos. Recordemos brevemente los textos.

IRC IV, 78 nos informa de que Quintus Calpurnius Nymphius, liberto de Quintus, fue seviro augustal y erigió el monumento funerario para él mismo y para su esposa, la seguramente también liberta, Marcia Vrania en un momento ubicable dentro de la segunda mitad del siglo I d.C.

IRC IV, 149, mucho más tardía ya que puede situarse a primeros del siglo III d.C., es un altar funerario dedicado por Quintus Calpurnius Oc\{e\}tavius a su contubernalis, Crescentina.

Finalmente, dos monumentos que ofrecen algunas dudas. El texto del primero, hoy perdido, nos ha llegado por un manuscrito de P.M. Anglès del siglo XVIII que conviene completar (IRC IV, 183), pero que nos deja abierta la puerta a la condición libre o liberta de la lista de personajes mencionados en nominativo, entre ellos una Calpurnia Fida, hija o liberta de Lucius. Por la noticia del lugar de descubrimiento, en el derribo de la

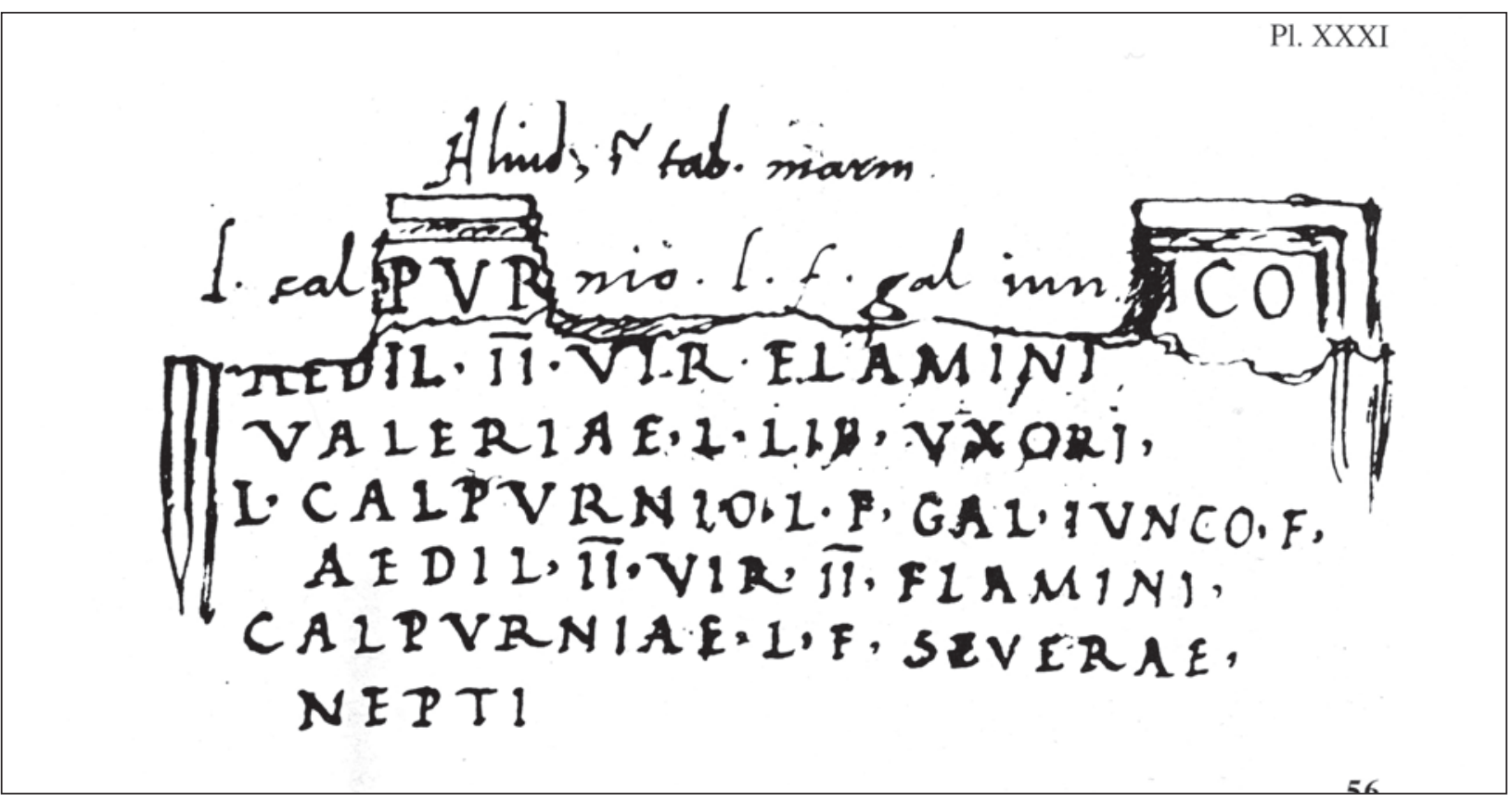

Figura 3.- IRC IV, 56 según M. Accursius, ms. Bibl. Ambrosiana de Milán, O 125 sup., fol. 79 ro . (s. XVI)

3 I. RODÀ, "La Barcelona de l'època romana 1. Etapa fundacional", III Congrés d'Història de Barcelona, Barcelona 1993, pp. 11-24; EAD., "La promoción de las elites en las ciudades del conventus Tarraconensis", Pluralidad e inte- gración en el mundo romano (Pamplona-Olite 2008), Actas del II Coloquio Internacional Italia Iberia - Iberia Italia F.J. Navarro, ed., Pamplona 2010, pp. 181-185. 
muralla romana, podría tratarse preferentemente de un monumento funerario.

En el segundo de los monumentos sólo podemos restituir hipotéticamente el nomen Calpurnius. Se trata de uno de los pedestales de la numerosa serie de los dedicados al influyente liberto Lucius Licinius Secundus (IRC IV, 92), hoy perdido, erigido a su óptimo amigo por dos libertos y seviros augustales de la colonia de Tarraco llamados los dos Marcus Galerius o Marcus Calpurnius (nos inclinaríamos a pensar probablemente que serían Calpurnios) que llevaban respectivamente los cognomina de Syrus y Gratus.

Fuera de Barcino, podemos mencionar que en la capital, Tarraco, son pocos los Calpurnios recordados por la epigrafía: dos de ellos, flámines provinciales y no nacidos en la ciudad ${ }^{4}$, y un tercero, con el cognomen de Tiro, que fue edil en el siglo I d.C. ${ }^{5}$ En la provincia de Barcelona hay un único Calpurnio, autor de la dedicatoria de un altar en Artès $(I R C \mathrm{I}, 25)$. En un cierto momento se pensó que quizás un Cal(purnius), hubiera podido ser uno de los productores de ánforas de Can Tintorer (El Papiol, Baix Llobregat), a juzgar por las marcas halladas (IRC V, 108), pero una revisión directa del material ha permitido descartar esta hipótesis ya que los sellos se leen como CALAM y no hay ninguna secuencia en las ánforas catalanas que pueda atribuirse a un Calpurnius ${ }^{6}$.

4 RIT, 264-265= CIL II2/14,2, 1124, 1125.

5 RIT, 340.

6 P. BERNI, C. CARRERAS, Barcino II: marques, terrisseries del Baix Llobregat, en prensa como un nuevo fascículo de la serie Corpus International des Timbres Amphoriques de la Unión Académica Internacional. Agradecemos a los autores las consultas sobre este trabajo suyo en proceso de edición.

7 Las excavaciones, dirigidas por Julia Miquel de la empresa Tea (código MUHBA 061/07), forman parte del proyecto "Análisis del comportamiento estructural, estudio de soluciones de consolidación y propuestas de intervención" en la iglesia de Santa Maria del Pi, según el convenio establecido por el Departamento de Cultura de la Generalitat de Cataluña, el Arzobispado de Cataluña y la propia parroquia. Hemos de agradecer a Eduard Riu y a Julia Miquel las facilidades para el acceso y estudio del pedestal, así como las
Al conjunto de inscripciones de los Calpurnii en Barcino, ha venido a sumarse recientemente el hallazgo de otro pedestal del que vamos a dar noticia a continuación y que nos permite establecer las relaciones que hubo, o pudo haber, entre ellos.

El pedestal (fig. 2) fue descubierto en 2007 en las excavaciones realizadas en la iglesia de Santa Maria del Pi, utilizado a principios del siglo XIV como material constructivo de la cimentación del paramento interno izquierdo de la portada ${ }^{7}$. Con posterioridad se procedió a su extracción ${ }^{8}$ y ahora se conserva en el recinto del templo romano, en la calle del Paradís (núm. de inventario MHCB 34884).

Es un pedestal moldurado de piedra caliza de Santa Tecla ${ }^{9}$, roto en su parte inferior a la altura de la última línea con pequeños golpes que afectan sobre todo a la moldura superior, asimismo el campo epigráfico presenta hendiduras y golpes que no dificultan la lectura pero si la visibilidad de la puntuación. En la parte superior se practicó una cavidad circular (11 $\mathrm{cm}$. de diámetro) como repositorio de reliquias, encajada en una oquedad cuadrangular para la tapa $(21 \times 18 \mathrm{~cm})$, lo que prueba que fue reutilizado como soporte de altar, a lo cual ha de deberse también el rebaje vertical de los dos ángulos posteriores del monumento. En el lateral izquierdo, líneas de juegos incisas formando un cuadrado con hueco central para la ficha (fig. 4), demuestran que el pedestal ya estaba caído en su lugar originario, seguramente el foro, cuando fue trasladado para ser reutilizado en la iglesia de Santa Maria del Pi.

que hemos hallado en el equipo del MUHBA y queremos mencionar explícitamente a Julia Beltrán y a Emili Revilla. Cf. las noticias sobre el hallazgo publicadas por J. BELTRÁN DE HEREDIA BERCERO, J. 2010. "Barcino, de colonia augustea a sede regia en época visigoda. Las transformaciones urbanas a la luz de las nuevas aportaciones de la arqueología", Arqueología, Patrimonio y desarrollo urbano. Problemática y soluciones. Girona 2010, pp. 3149, esp. p. 44, fig.10, y también por T. JUÁREZ, "Església de Santa Maria del Pi", Anuari d'Arqueologia i Patrimoni de Barcelona 2008, Barcelona 2010, p. 55.

8 La intervención, con el código 137/07, fue dirigida por T. Juárez de la empresa Tea. Cf. nota anterior.

9 Sobre este material lapídeo, v. A. ÀLVAREZ, V. GARCÍAENTERO, A. GUTIÉRREZ, I. RODÀ, El marmor de Tarraco/Tarraco Marmor, Hic et Nunc 6, ICAC, Tarragona 2009. 


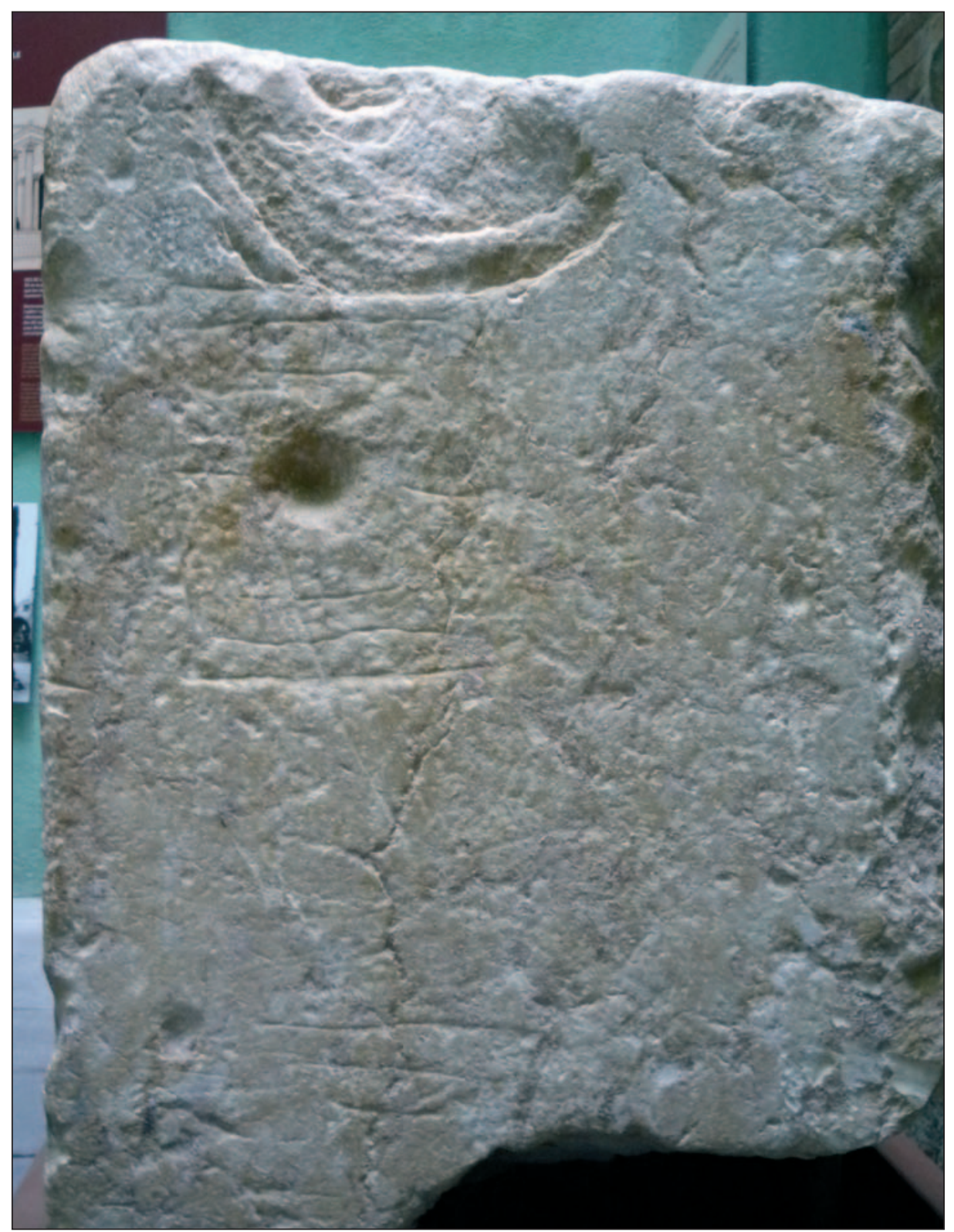

Figura 4.- Tablero de juego inciso en el lateral izquierdo del pedestal hallado en la iglesia de Santa Maria del Pi. Foto I. Rodà.

Medidas totales: 67 máx x 57 x 54 .

Campo epigráfico: 59,5 máx x 47.

Altura de las letras: $6(N I: 8 ; O: 1,8) ; 5,4 ; 5,1 ; 4,5 ; 4,5(T: 5,3)$; c.4.

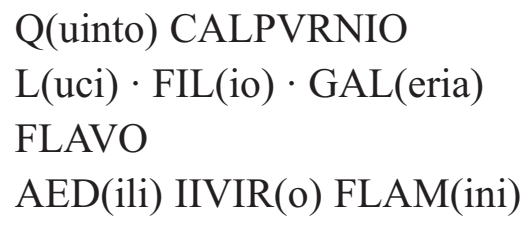

$5 \quad$ ROM(ae) ET AVG(usti)

$[$ BARC]IN(onenses) $\cdot \mathrm{EX} \cdot \mathrm{D}$ (ecurionum) $\mathrm{D}$ (ecreto) 
A Quinto Calpurnio Flavo, hijo de Lucio de la tribu Galeria, edil, duumviro, flamen de Roma y del Augusto. Los Barcinonenses por decreto de los decuriones.

Letra capital cuadrada, bien grabada y muy regular. $O$ minuta en 1 . 1 . Nexo $N I$ en 1 . 1. Puntuación poco perceptible en forma de espina de rosa en las líneas 2 y 6 y ausencia de puntos al final de las líneas. Ordinatio sencilla y bien equilibrada, centrando bien las líneas, amplia separación entre las 1, 5 y la última, aunque cabe destacar un mal cálculo en la distribución de las letras de 1. 1 (nexo final y $O$ minuta) y en 1.4 con la $M$ dispuesta sobre la moldura.

El personaje pasó por todos los cargos municipales de Barcino, culminando su carrera como flamen Romae et Augusti que también desempeñó en la ciudad Lucius Mandulius Crescens a finales del siglo I o comienzos del II quien, después de este honos, saltó a la categoría ecuestre como praefectus fabrum (IRC IV, 42). También obtuvo este cargo, con la duda de si podía haber sido flamen Romae et Augusti o Augustorum debido al carácter fragmentario del monumento, Manius Acilius Firmanus por unos mismos años, por lo que creemos más probable que hubiera ejercido como sacerdote del Augusto y no de los Augustos (IRC IV, 50).

Nuestro Cornelio Flavo cumplió satisfactoriamente sus funciones, según nos deja suponer la dedicatoria que restituimos como de los Barcinonenses por decreto de los decuriones. Nos parece que no hay el espacio suficiente para la fórmula $R P B A R C \mathrm{y}$, caso de desarrollar Barcinonensium en lugar de Barcinonenses, lo lógico es pensar que hubiera precedido la fórmula $E X D D$, como vemos por ejemplo en IRC IV 84-88.

Este nuevo pedestal podría datarse en el último cuarto del siglo I d.C. y nos permite establecer unas relaciones, en unos casos seguras y en otros probables. En primer lugar la homonimia del personaje homenajeado en

10 I. KAJANTO, Latin Cognomina, Helsinki 1965, reed. Roma 1982, p. 334 recoge que fue llevado por 5 senadores; A. MÓCSY, Nomenclator provinciarum Europae, el nuevo texto con el conservado en el restaurante de la Torre dels Lleons en Esplugues de Llobregat (IRC IV, 55, fig. 1), pero de los dos Quintos Calpurnios Flavos, uno es hijo de Lucio y el otro de Quinto.

Por lo que se refiere al texto de $I R C$ IV, 56 (fig. 3), el buen cálculo del espacio disponible y la onomástica del hijo llevaron a Accursius a restituir en la primera línea el praenomen Lucius de [Cal]pur[nius] [Iu]ncus, edil, duumvir y flamen y padre también de Lucio Calpurnio Junco, asimismo edil, duumvir por dos veces y flamen.

Comprobamos que los Calpurnios magistrados recordados en estas tres inscripciones, o bien llevaron el praenomen Lucius o Quintus, así como la repetición de una misma onomástica entre los miembros de la familia y la continuidad de padres a hijos en el ejercicio de las carreras municipales, conformando unos "lobbies" que cada vez son mejor conocidos dentro de la sociedad barcinonense.

Podríamos proponer, teniendo en cuenta también la cronología de los monumentos, que Lucius Calpurnius Iuncus, con un cognomen poco frecuente de connotaciones aristocráticas $^{10}$ que sólo aparece en Hispania en esta inscripción de padre del homónimo iunior, fuera el iniciador de la saga barcinonense.

De uno $\mathrm{u}$ otro de estos personajes podría muy bien haber sido hijo el nuevo Quinto Calpurnio Flavo (fig. 2), la onomástica del cual nos parece a su vez que hace evidente la paternidad del magistrado de IRC IV, 55 (fig. 1) llamado asimismo Quinto Calpurnio Flavo, hijo de Quinto, muerto a los 29 años, edil, duumviro y al que el ordo barcinonense le concedió post mortem los honores flaminales. Como quien dedica el pedestal es su madre, Cominia Nereis, hemos de suponer que ésta fue la esposa del nuevo Calpurnio Flavo.

Llegados a este punto, podemos establecer una constatación tipológica interesante. El pedestal de Quinto Calpurnio Flavo padre, presenta un texto que se centra en la mitad

Dissertationes Pannonicae III, 1, Budapest 1983, p. 155; B. LÖRINCZ, Onomasticon provinciarum Europae Latinarum, vol. II, Viena 1999, p. 207. 
superior del campo epigráfico, con una letra capital de forma más cuadrada. En cambio, el pedestal de Calpurnio hijo presenta una letra más alargada y el texto ocupa un mayor espacio. Si comparamos estos dos pedestales con los de otra gens de Barcino que tuvo un importante papel, la Trocina, el paralelo surge de manera natural. De los tres pedestales conocidos, dos (IRC IV 110 y 112) tienen una distribución parecida a la de la inscripción de Calpurnio padre, con un gran espacio inferior en blanco. Este tipo habría podido salir del taller epigráfico dentro del último cuarto del siglo I d.C., en época flavia. En cambio el pedestal de Calpurnio hijo se podría poner en paralelo con IRC IV 111, forzosamente posterior a $I R C$ IV 110 , y con un texto más largo que se expande por el campo epigráfico; este tipo podría irse ya a los años finales del siglo I d.C. y primeros del II.

Por lo que a los Calpurnios libertos respecta, además de las esposas, y una de ellas madre, de los magistrados municipales, el Quinto Calpurnio Ninfio mencionado en IRC IV, 78, de una cronología similar a la de $I R C$ IV, 56 y a la del nuevo pedestal, bien hubiera podido ser el liberto de Quinto Calpurnio Flavo, hijo de Lucio.

Por otra arte, un recuerdo más tardío de un Quintus Calpurnius lo tenemos en IRC IV, 149, que nos atestigua la continuidad de la familia en Barcino y del praenomen Quintus todavía a inicios del siglo III d.C., pero poco más nos permite aportar.
El hallazgo del nuevo pedestal en Barcino nos ha permitido profundizar en el conocimiento de su sociedad en época altoimperial y comprobar una vez más la importancia de ciertas familias de la aristocracia municipal en la sucesión de padres a hijos en el ejercicio de las magistraturas. También el carácter abierto del marco social, con un entronque con personajes de condición libertina y una continuidad de la gens mediante los esclavos manumitidos que pudieron promocionarse con el ejercicio del sevirato augustal dentro de la potente institución del culto imperial, auténtico motor de integración y movilidad en la política de los siglos I y II d.C., época de gran vitalidad para la colonia de Barcino.

\section{BIBLIOGRAFIA:}

CIL II2/14, 2 = G. ALFÖLDY, Corpus Inscriptionum Latinarum editio altera, II, 14, fasc. 2. Colonia Iulia Urbs Triumphalis Tarraco, Berlín-Nueva York 2011.

$I R C \mathrm{I}=\mathrm{G}$. FABRE, M. MAYER, I, RODÀ, Inscriptions romaines de Catalogne I. Barcelone (sauf Barcino), París 1984.

$I R C \mathrm{IV}=\mathrm{G}$. FABRE, M. MAYER, I, RODÀ, Inscriptions romaines de Catalogne IV. Barcino, París 1997.

IRC $\mathrm{V}=\mathrm{G}$. FABRE, M. MAYER, I, RODÀ, Inscriptions romaines de Catalogne $V$. Suppléments aux volumes I-IV et instrumentum inscriptum, París 2002.

RIT $=\mathrm{G}$. ALFÖLDY, Die römischen Inschriften von Tarraco (Madrider Forschungen 10), Berlín 1975. 
